\title{
Application of Closed Queuing Network to Computer System
}

\author{
${ }^{1}$ Agboola S. O. and ${ }^{2}$ Ayinde S. A. \\ ${ }^{I}$ Department of Mathematics with Applied Statistics, Bells University of Technology, Ota, Ogun State, Nigeria. \\ ${ }^{2}$ Department of Basic Sciences, Babcock University, Ilisan Remo, Ogun State, Nigeria
}

\begin{abstract}
A closed queuing network that consists of $M$ nodes was studied, and in contrast to an open network, there is a constant population of $K$ customers in the network with no external source or sink. Each node $i$ is a first in first out queue, where the service time is drawn independently. That is, the service time is exponentially distributed with service rates $\mu_{i}\left(n_{i}\right)$ and a customer departing from queue $i$ chooses $j$ next with probability $q_{i, j}$. The Gordon and Newel theorem with the arrival properties of a closed network were used to analyze the Mean values for the number of customers, sojourn time, waiting times and the algorithm for solving the mean value for closed networks were then derived with series of application considered.
\end{abstract}

\section{Introduction}

Queuing network models have been seen as the cost effective tools for analyzing modern and multi programmed systems and can also be used for calculating and predicting performance quantities such as utilizations, mean queue lengths, mean response times and operational analysis among them.

In 1957, Jackson published an analysis of a multiple device system such that each device contained one or more parallel servers and jobs could enter or exit the system anywhere.

In 1963, Jackson extended his analysis to open and closed network systems with local load dependent service rate at all device.

In 1965, Scherr carried out the first successful application of a network model to a computer system, when he used the classical machine repairman model to analyze the MIT time sharing system.

In 1967, Gordon and Newel simplified the notational structured of these results for the special case of closed system.

In 1971, Buzen introduced the central server model and Moore in the same year showed that queuing network models could predict the response times on the Michigan Terminal system (MTS) to within $10 \%$. The extensive validation since 1971 have verified that these models reproduce observed accuracy and the traditional approach to deriving the accuracy of queuing network results depends on a series of assumptions used in the theory of stochastic processes such as Job are stochastically independent, job steps from device to device follows a Markov chain, the system is stochastically equilibrium, the system is ergodic and the service time requirements at each device conform to an exponential distribution.

\section{NOTATIONS}

$\mathrm{a} / \mathrm{b} / \mathrm{c} \quad$ Queue Short - hand Notation

$q_{i, j} \quad$ The Probability that upon departure from queue $i$, the customers chooses the next queue $j$

$P(n)=P\{N=n\} \quad$ The state probability

$S \quad$ Source or External

$d \quad$ Destination or Sink

$\bar{T} \quad$ Mean Sojourn Time of nth customers

$\lambda \quad$ Arrival Rate, Intensity and Parameter of Distribution

$\lambda_{i} \quad$ Average customer flow through node $i$

$N \quad$ Queue lengths or number of customers in the system

$N_{i} \quad$ Number of customers in node $i$

$\overline{N_{i}} \quad$ Mean queue length or mean number of customers in node $i$

$\overline{W_{i}} \quad$ Mean waiting time in node $i$

$\rho \quad$ Traffic Workload and Occupational Rate 


$\begin{array}{ll}\mu_{i} & \text { Service rate at node } i \\ H & \text { Lagrange's Multiplier } \\ C & \text { Cost Constraint } \\ \alpha & \text { Solution on closed network } \\ \wedge & \text { The value of } \alpha \text { that correspond to the actual streams which remain } \\ \alpha & \text { Undetermined } \\ K & \text { The constant population of customers in the closed network } \\ M & \text { Number of nodes in the closed network }\end{array}$

\section{Materials And Method}

In this study, a customer departing from queue $i$ chooses queue $j$ next with probability $q_{i, j}$ and the customer streams through different nodes satisfy the conservation law

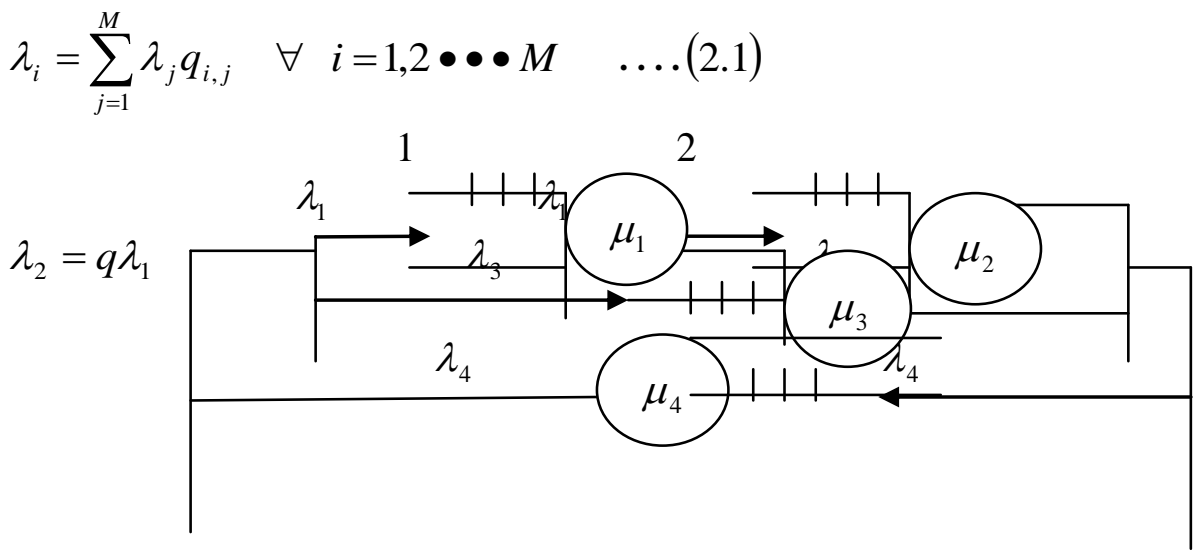

\section{TRANSITION DIAGRAM FOR CLOSED QUEUEING NETWORK}

Equations $(i)$ constitute a homogenous linear set of equations such that one equation is linearly dependent on the others, and the solution is determined uniquely up to a constant factor.

Let $\left(\hat{\lambda}_{1}, \bullet \bullet \bullet, \hat{\lambda}_{M}\right)$ be a solution. The general solution is of the form $\alpha \bullet\left(\hat{\lambda}_{1}, \bullet \bullet \bullet, \hat{\lambda}_{M}\right)$, where $\alpha$ is a constant and the value of $\alpha$ corresponds to the actual streams remain undetermined. If this value be denoted by $\hat{\alpha}$, i.e. $\lambda_{i}=\hat{\alpha} \hat{\lambda}_{i}$ and $\hat{\rho}_{i}=\frac{\hat{\lambda}_{i}}{\mu_{i}}$ which are correspondingly proportional to the real loads of the queues $\rho_{i}=\frac{\lambda_{i}}{\mu_{i}}$ such that $\rho_{i}=\hat{\alpha} \hat{\rho}_{i}$

The equilibrium probabilities of a closed queueing network are

$$
p(n)=\left\{\begin{array}{lr}
\frac{1}{G(K, M)} \prod_{i=1} \hat{\rho}_{i}^{n_{i}} & \text { when } \sum_{\mathrm{i}} n_{i}=K \\
0 & \text { when } \sum_{\mathrm{i}} n_{i} \neq K
\end{array}\right.
$$

Where $G(K, M)=\sum_{n: \sum_{i} n_{i}=K} \prod_{i=1}^{M} \hat{\rho}_{i}^{n_{i}}$ 


\subsection{Arrival Theorem in Closed Networks}

In a closed network with $\mathrm{K}$ customers, the state probabilities seen by a customer entering any node are the same as the equilibrium probabilities $p[K-1](n)$ in a network with $[K-1]$ customers. This shows that the arbitrary customer is as if he were an external observer.

\subsection{Mean Value Analysis of Closed Networks}

The main aim of mean value analysis is to find the mean number of customers $\bar{N}_{i}[K]$ and sojourn time $\overline{T_{i}}[K]$ as well as the absolute values of customer streams $\lambda_{i}$ through different queues.

The analysis is centrally based on the arrival theorem. The calculation proceeds recursively, incrementing the customer population in the network step by step.

The mean sojourn time in queue $i$ is

$\overline{T_{i}}[K]=\frac{1}{\mu_{i}}+\overline{N_{i}^{*}}[K] \bullet \frac{1}{\mu_{i}}$

Where $\frac{1}{\mu_{i}}$ is the service time in queue $i, \overline{N_{i}^{*}}[K] \bullet \frac{1}{\mu_{i}}$ is the time it takes to serve the customers ahead and $\overline{N_{i}^{*}}[K]$ is the mean occupancy seen by a customer arriving at queue $i$.

By the arrival theorem

$\overline{N_{i}^{*}}[K]=\overline{N_{i}^{*}}[K-1]$

Where $\overline{N_{i}{ }^{*}}[K-1]$ is the mean occupancy calculated from the equilibrium distribution. Thus $\overline{T_{i}}[K]=\left[1+\overline{N_{i}^{*}}[K]\right] \cdot \frac{1}{\mu_{i}}$

The mean occupancy in queue $i$ is $\overline{N_{i}^{*}}[K]=K \bullet \frac{\hat{\lambda}_{i} \bullet \bar{T}_{i}[K]}{\sum_{j=1}^{M} \hat{\lambda}_{j} \bullet \bar{T}_{j}[K]}$

Proof:

The real customer streams are $\lambda_{i}=\hat{\alpha} \hat{\lambda}_{i}$. By expanding the above expression by $\hat{\alpha}$ and by Little's result we have,

$$
K \bullet \frac{\hat{\lambda}_{i} \bullet \bar{T}_{i}[K]}{\sum_{j=1}^{M} \hat{\lambda}_{j} \bullet \bar{T}_{j}[K]}=K \bullet \frac{\lambda_{i}[K] \bullet \bar{T}_{i}[K]}{\sum_{j=1}^{M} \lambda_{j}[K] \bullet \bar{T}_{j}[K]}=K \bullet \frac{\overline{N_{i}}[K]}{\sum_{j=1}^{M} \overline{N_{j}}[K]}=K \bullet \frac{\overline{N_{i}}[K]}{K}=\overline{N_{i}}[K]
$$

By using Little's result in the reverse direction, the real customer stream through queue $i$ is

$$
\lambda_{i}[K]=\frac{\bar{N}_{i}[K]}{T_{i}[K]}=K \bullet \frac{\hat{\lambda}_{i}}{\sum_{j=1}^{M} \hat{\lambda}_{j} \bar{T}_{j}[K]} .
$$

By recursive process, the mean value algorithm for closed queueing network can be obtained as follows:

Set $\bar{N}_{i}[0]=0$

$$
\begin{aligned}
& \overline{T_{i}}[K]=\left(1+\overline{N_{i}}[K-1]\right) \times \frac{1}{\mu_{i}} \\
& \overline{N_{i}}[K]=K \bullet \frac{\hat{\lambda}_{i} \bullet \bar{T}_{i}[K]}{\sum_{j=1}^{M} \hat{\lambda}_{j} \bullet \bar{T}_{j}[K]}
\end{aligned}
$$


$\lambda_{i}[K]=\frac{\bar{N}_{i}[K]}{T_{i}[K]}$

Where the $\hat{\lambda}_{i}$ are any solution to the flow equations $\lambda_{i}=\sum_{j} \lambda_{j} q_{j, i}$.

\subsection{Practical Example 1:}

Given the following closed system as follows

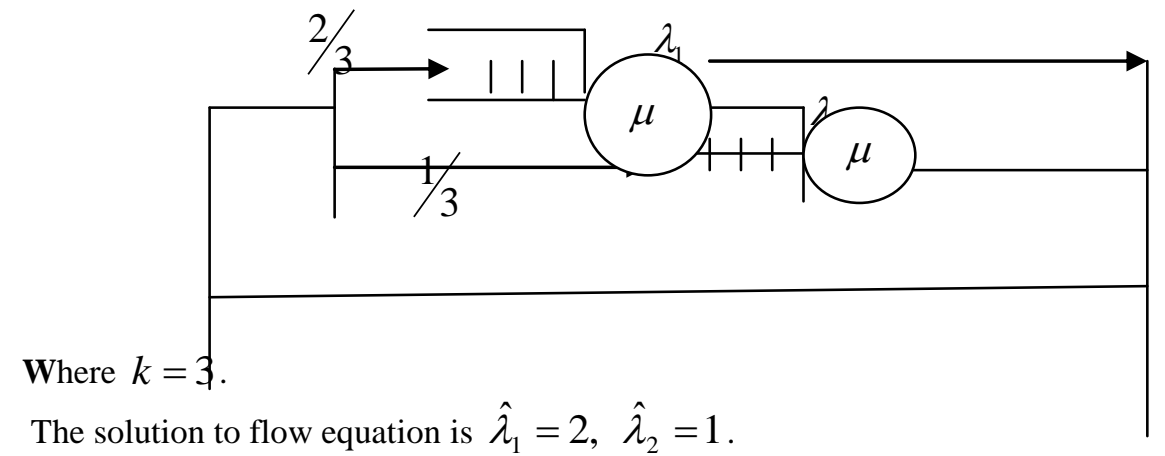

Starting from

$$
\bar{N}_{1}[0]=\bar{N}_{2}[0]=0
$$

The mean values for progressively greater populations can be solved

$$
\begin{aligned}
& K=1 \quad\left\{\begin{array} { l } 
{ \overline { \mathrm { T } } _ { 1 } [ 1 ] = \frac { 1 } { \mu } } \\
{ \overline { \mathrm { N } } _ { 1 } [ 1 ] = 1 \bullet \frac { 2 / \mu } { 2 / \mu + \frac { 1 } { \mu } } = \frac { 2 } { 3 } } \\
{ \lambda _ { 1 } [ 1 ] = \frac { 2 } { 3 } \mu }
\end{array} \left\{\begin{array}{l}
\overline{\mathrm{T}}_{2}[1]=\frac{1}{\mu} \\
\overline{\mathrm{N}}_{2}[1]=1 \bullet \frac{1 / \mu}{2 / \mu+\frac{1}{\mu}}=\frac{1}{3} \\
\lambda_{2}[1]=\frac{1}{3} \mu
\end{array}\right.\right. \\
& K=2\left\{\begin{array} { l } 
{ \overline { \mathrm { T } } _ { 1 } [ 2 ] = ( 1 + \frac { 2 } { 3 } ) \frac { 1 } { \mu } = \frac { 5 } { 3 } \frac { 1 } { \mu } } \\
{ \overline { \mathrm { N } } _ { 1 } [ 2 ] = 2 \cdot \frac { 2 \cdot 5 / 3 } { 2 \cdot 5 / 3 + 1 \cdot 4 / 3 } = \frac { 1 0 } { 7 } } \\
{ \lambda _ { 1 } [ 2 ] = \frac { 6 } { 7 } \mu }
\end{array} \left\{\begin{array}{l}
\overline{\mathrm{T}}_{2}[2]=\left(1+\frac{1}{3}\right) \frac{1}{\mu}=\frac{4}{3} \frac{1}{\mu} \\
\overline{\mathrm{N}}_{2}[2]=2 \cdot \frac{1 \cdot 4 / 3}{2 \cdot 5 / 3+1 \cdot 4 / 3}=\frac{4}{7} \\
\lambda_{2}[2]=\frac{3}{7} \mu
\end{array}\right.\right. \\
& K=3\left\{\begin{array} { l } 
{ \overline { \mathrm { T } } _ { 1 } [ 3 ] = ( 1 + \frac { 1 0 } { 7 } ) \frac { 1 } { \mu } = \frac { 1 7 } { 7 } \frac { 1 } { \mu } } \\
{ \overline { \mathrm { N } } _ { 1 } [ 3 ] = 3 \cdot \frac { 2 \cdot 1 7 / 7 } { 2 \cdot 1 7 / 7 + 1 \cdot 1 1 / 7 } = \frac { 3 4 } { 1 5 } } \\
{ \lambda _ { 1 } [ 3 ] = \frac { 1 4 } { 1 5 } \mu }
\end{array} \left\{\begin{array}{l}
\overline{\mathrm{T}}_{2}[3]=\left(1+\frac{4}{7}\right) \frac{1}{\mu}=\frac{11}{7} \frac{1}{\mu} \\
\overline{\mathrm{N}}_{2}[3]=3 \cdot \frac{1 \cdot 11 / 7}{2 \cdot 17 / 7+1 \cdot 11 / 7}=\frac{11}{15} \\
\lambda_{2}[3]=\frac{7}{15} \mu
\end{array}\right.\right.
\end{aligned}
$$


EXAMPLE 2:

Given the cyclic network as shown below:

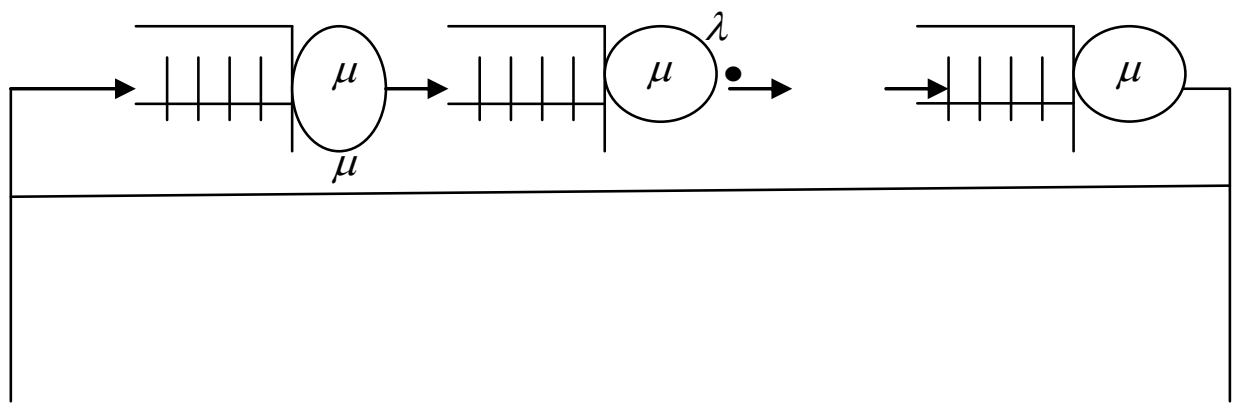

Such that $\mu_{1}=\mu_{2}=\mu_{3}=\bullet \bullet \bullet=\mu_{M}=\mu$.

A solution to the flow equations is:

$\hat{\lambda}_{1}=\hat{\lambda}_{2}=\hat{\lambda}_{3}=\bullet \bullet \bullet=\hat{\lambda}_{M}=1$.

Since all the queues are identical, the recursion equations are as follows:

$\left\{\begin{array}{l}\bar{T}[K]=(1+\bar{N}[K-1]) \times \frac{1}{\mu} \\ \bar{N}[K]=K / M \\ \lambda[K]=\bar{N}[K] / \bar{T}[K]\end{array}\right.$

By setting $\bar{N}[0]=0$, the mean values for progressively greater populations can be solved as follows:

$\left\{\begin{array}{l}\bar{T}[1]=\frac{1}{\mu} \\ \bar{N}[1]=1 / M \\ \lambda[1]=\frac{1}{M} \mu\end{array} \quad\left\{\begin{array}{l}\bar{T}[2]=\frac{M+1}{M \mu} \\ \bar{N}[2]=2 / M \\ \lambda[2]=\frac{2}{M+1} \mu\end{array} \quad\left\{\begin{array}{l}\bar{T}[3]=\frac{M+2}{M \mu} \\ \bar{N}[3]=3 / M \\ \lambda[3]=\frac{3}{M+2} \mu\end{array} \bullet \bullet\left\{\begin{array}{l}\bar{N}[K]=K / M \\ \lambda[K]=\frac{K}{M+K-1} \mu\end{array}\right.\right.\right.\right.$

When $K<M$ then $\lambda[K] \approx K / M \mu$ (This implies that the mean time of full cycle is $M / \mu$ when there are $K$ customers.

When $K>M$ then $\lambda[K] \approx \mu$ (This implies that customers depart on average at intervals $1 / \mu$ when the queues are full).

\section{Conclusion:}

The closed queueing networks was operationally analyzed with the help of flow or transition diagram, conservation law and arrival theorem to arrived at the mean values for sojourn time, number of customers and the absolute values of customer streams $\lambda_{i}$ through different queues. The recursive algorithms for the aforementioned parameters were later derived with the numerical application demonstrated on closed and cyclic networks.

\section{References}

[1]. Agboola, S.O. (2007): On the Analysis of M/G/1 Queues. Unpublished M.Sc. (Mathematics) Thesis submitted to Department of Mathematics, University of Ibadan.

[2]. Agboola, S.O. (2011): The Analysis of Markov Inter-arrival Queues Model with K - Server under Various Service Points. Unpublished M.Sc. (Statistics) Thesis submitted to Department of Mathematics, Obafemi Awolowo University, Ile-Ife.

[3]. Balbo, G.; Bruell, S. C.; and Schwetman, H. D. (1977): "Customer classes and closed network models - a solution techniques," in Proc. IFIP Congress 77, North - Holland Publ. Co., Amsterdam, The Netherland. 
[4]. Baskett, F.; Chandy, K. M.; Muntz, R. R.; and Palacios, J. (1975): Open, Closed and Mixed Networks with Different Classes of Customers. J. ACM 22, 2. pp $248-260$.

[5]. Buzen, J. P. (1971): Queueing Network Models of Multiprogramming, PhD Thesis, Div. Eng. And Applied Physics, Harvard University.

[6]. Buzen, J. P. (1973): Computational Algorithms for Closed Queueing Networks with Exponential Servers. Commun. ACM 16, 9, pp $527-531$.

[7]. Chang, A.; and Lavenberg, S. (1974): Work Rate in Closed Queueing Networks with General Independent servers, Oper. Res. 22, 4, pp $838-847$.

[8]. Chandy, K. M.; Herzog, U.; and Woo, L. (1975): Parametric Analysis of Queueing Networks, IBM J Res Dev. 19, 1. pp 36 - 42.

[9]. Chandy, K. M.; Herzog, U.; and Woo, L. (1975): Approximate Analysis of General Queueing Networks, IBM J Res Dev. 19, 1, pp $43-59$.

[10]. Denning, P. J.; and Buzen, J. P. (1977): Operational Analysis of Queueing Networks, In Proc. Third Int. Symp. Computer Performance Modeling, Measurement and Evaluation, North - Holland Publ. Co., Amsterdam, The Netherland.

[11]. Gordon, W. J.; and Newell, G. F. (1967): Closed Queueing System with Exponential Servers, Oper. Res. 15, pp 254 - 265.

[12]. Jackson, J. R. (1957): Networks of Waiting Lines, Oper. Res. 5, pp $518-521$.

[13]. Kleinrock, L. (1975): Queueing Systems I, John Wiley, New York.

[14]. Kleinrock, L. (1976): Queueing Systems II, John Wiley, New York.

[15]. Reiser, M.; and Kobayshi, H. (1975): Queueing Networks with Multiple Closed Chains, Theory and Computation Algorithms, IBM J. Res. Dev. 19, pp $283-294$.

[16]. Schum, A. W. C.; (1976): Queueing Models for Computer System with General Service time Distributions, PhD Thesis, Div. Eng. And Applied Physics, Harvard University, Cambridge, Mass. 